\title{
Remarks on Ultrametrics and Metric-Preserving Functions
}

\author{
Prapanpong Pongsriiam ${ }^{1}$ and Imchit Termwuttipong ${ }^{2}$ \\ ${ }^{1}$ Department of Mathematics, Faculty of Science, Silpakorn University, Nakhon Pathom 73000, Thailand \\ ${ }^{2}$ Department of Mathematics and Computer Science, Faculty of Science, Chulalongkorn University, Bangkok 10330, Thailand
}

Correspondence should be addressed to Prapanpong Pongsriiam; prapanpong@gmail.com

Received 22 December 2013; Accepted 19 April 2014; Published 30 April 2014

Academic Editor: Gaston M. N’Guérékata

Copyright (C) 2014 P. Pongsriiam and I. Termwuttipong. This is an open access article distributed under the Creative Commons Attribution License, which permits unrestricted use, distribution, and reproduction in any medium, provided the original work is properly cited.

Functions whose composition with every metric is a metric are said to be metric-preserving. In this paper, we investigate a variation of the concept of metric-preserving functions where metrics are replaced by ultrametrics.

\section{Introduction}

Under what conditions on a function $f:[0, \infty) \rightarrow[0, \infty)$ is it the case that for every metric space $(X, d), f \circ d$ is still a metric? It is well known that for any metric $d, d /(1+d)$ and $\min \{1, d\}$ are bounded metrics topologically equivalent to $d$, while $d /\left(1+d^{2}\right)$ need not be a metric.

We call $f:[0, \infty) \rightarrow[0, \infty)$ metric-preserving if for all metric spaces $(X, d), f \circ d$ is a metric. Therefore, the functions $f$ and $g$ given by $f(x)=x /(1+x)$ and $g(x)=\min \{1, x\}$ are metric-preserving but $h(x)=x /\left(1+x^{2}\right)$ is not. The concept of metric-preserving functions first appears in Wilson's article [1] and is thoroughly investigated by many authors; see for example, [2-18] and references therein.

However, other important types of distances such as ultrametrics, pseudometrics, pseudodistances $[19,20], w$ distances, and $\tau$-distances have not yet been developed in the connection with metric-preserving functions. These distances have many applications in mathematics; see, for example, applications of $w$-distances and $\tau$-distances in [2127]. We will particularly be concerned with the ultrametrics which arise naturally in the study of $p$-adic numbers and nonarchimedean analysis $[28,29]$, topology and dynamical system [30-33], topological algebra [34], and theoretical computer science [35].

In connection with ultrametrics and metric-preserving functions, the problem arises to investigate the properties of the following functions and compare them with those of metric-preserving functions.
Definition 1. Let $f:[0, \infty) \rightarrow[0, \infty)$. We say that

(i) $f$ is ultrametric-preserving if for all ultrametric spaces $(X, d), f \circ d$ is an ultrametric;

(ii) $f$ is metric-ultrametric-preserving if for all metric spaces $(X, d), f \circ d$ is an ultrametric;

(iii) $f$ is ultrametric-metric-preserving if for all ultrametric spaces $(X, d), f \circ d$ is a metric.

For convenience, we also let $\mathscr{M}$ be the set of all metricpreserving functions, $\mathcal{U}$ the set of all ultrametric-preserving functions, $\mathcal{U} \mathscr{M}$ the set of all ultrametric-metric-preserving functions, and $\mathscr{M} \mathscr{U}$ the set of all metric-ultrametricpreserving functions.

We will give some basic definitions and useful results that will be used throughout this paper in the next section. We then give properties and characterizations of those functions in Sections 3, 4, and 5. We discuss and give some results on the continuity aspect of those functions in Section 6 .

\section{Preliminaries and Lemmas}

In this section, we give some basic definitions and results for the convenience of the reader. First, we recall the definition of a metric space and an ultrametric space.

A metric space is a set $X$ together with a function $d: X \times$ $X \rightarrow[0, \infty)$ satisfying the following three conditions:

(M1) For all $x, y \in X, d(x, y)=0$ if and only if $x=y$, 
(M2) for all $x, y \in X, d(x, y)=d(y, x)$, and

(M3) for all $x, y, z \in X, d(x, y) \leq d(x, z)+d(z, y)$.

An ultrametric space is a metric space $(X, d)$ satisfying the stronger inequality (called the ultrametric inequality):

(U3) for all $x, y, z \in X, d(x, y) \leq \max \{d(x, z), d(z, y)\}$.

A metric space $(X, d)$ is said to be topologically discrete if for every $x \in X$ there is an $\varepsilon>0$ such that $B_{d}(x, \varepsilon)=\{x\}$, where $B_{d}(x, \varepsilon)$ denote the open ball center at $x$ and radius $\varepsilon$. In addition, $(X, d)$ is said to be uniformly discrete if there exists an $\varepsilon>0$ such that $B_{d}(x, \varepsilon)=\{x\}$ for every $x \in X$.

Next we recall the definitions concerning certain behaviors of functions. Throughout, we let $f:[0, \infty) \rightarrow[0, \infty)$ and let $I \subseteq[0, \infty)$. Then $f$ is said to be increasing on $I \subseteq$ $[0, \infty)$ if $f(x) \leq f(y)$ for all $x, y \in I$ satisfying $x<y$, and $f$ is said to be strictly increasing on $I \subseteq[0, \infty)$ if $f(x)<f(y)$ for all $x, y \in I$ satisfying $x<y$. The notion of decreasing or strictly decreasing functions is defined similarly.

The function $f$ is said to be amenable if $f^{-1}(\{0\})=\{0\}$, and $f$ is said to be tightly bounded on $(0, \infty)$ if there is $v>$ 0 such that $f(x) \in[v, 2 v]$ for all $x>0$. We say that $f$ is subadditive if $f(a+b) \leq f(a)+f(b)$ for all $a, b \in[0, \infty), f$ is convex if $f\left((1-t) x_{1}+t x_{2}\right) \leq(1-t) f\left(x_{1}\right)+t f\left(x_{2}\right)$ for all $x_{1}, x_{2} \in[0, \infty)$ and $t \in[0,1]$, and $f$ is concave if $f\left((1-t) x_{1}+\right.$ $\left.t x_{2}\right) \geq(1-t) f\left(x_{1}\right)+t f\left(x_{2}\right)$ for all $x_{1}, x_{2} \in[0, \infty)$ and $t \in$ $[0,1]$. As mentioned earlier, we say that $f$ is metric-preserving if for all metric spaces $(X, d), f \circ d$ is a metric. Furthermore, $f$ is strongly metric-preserving if $f \circ d$ is a metric equivalent to $d$ for every metric $d$.

Now we are ready to state the results which will be applied in the proof of our theorems.

Lemma 2. Let $f:[0, \infty) \rightarrow[0, \infty)$. If $f$ is amenable, subadditive, and increasing on $[0, \infty)$, then $f$ is metricpreserving.

Proof. The proof can be found, for example, in $[4,6]$.

Lemma 3. If $f:[0, \infty) \rightarrow[0, \infty)$ is amenable and tightly bounded, then $f$ is metric-preserving.

Proof. The proof can be found, for example, in $[3,4]$.

The next lemma might be less well known, so we give a proof here for completeness.

Lemma 4. If $f:[0, \infty) \rightarrow[0, \infty)$ is amenable and concave, then the function $x \mapsto f(x) / x$ is decreasing on $(0, \infty)$.

Proof. Let $a, b \in(0, \infty)$ and $a<b$. Since $f$ is concave, we obtain

$$
\begin{aligned}
f(a) & =f\left(\left(1-\frac{a}{b}\right)(0)+\left(\frac{a}{b}\right)(b)\right) \\
& \geq\left(1-\frac{a}{b}\right) f(0)+\frac{a}{b} f(b) \\
& =\frac{a}{b} f(b) .
\end{aligned}
$$

Therefore, $f(a) / a \geq f(b) / b$, as desired.
Lemma 5. Let $(X, d)$ be an ultrametric space. Then for every $x_{1}, x_{2}, \ldots, x_{n} \in X$,

$$
d\left(x_{1}, x_{n}\right) \leq \max \left\{d\left(x_{1}, x_{2}\right), d\left(x_{2}, x_{3}\right), \ldots, d\left(x_{n-1}, x_{n}\right)\right\} .
$$

Proof. We have

$$
\begin{aligned}
d\left(x_{1}, x_{n}\right) & \leq \max \left\{d\left(x_{1}, x_{2}\right), d\left(x_{2}, x_{n}\right)\right\} \\
& \leq \max \left\{d\left(x_{1}, x_{2}\right), \max \left\{d\left(x_{2}, x_{3}\right), d\left(x_{3}, x_{n}\right)\right\}\right\} \\
& =\max \left\{d\left(x_{1}, x_{2}\right), d\left(x_{2}, x_{3}\right), d\left(x_{3}, x_{n}\right)\right\} .
\end{aligned}
$$

A repeated application of the ultrametric inequality as above gives the desired result.

Next we give basic relations and properties of the functions in $\mathscr{M}, \mathscr{U}, \mathscr{M} \mathscr{U}$, and $\mathcal{U} \mathscr{M}$.

Proposition 6. The following relations hold $\mathscr{M} \mathcal{U} \stackrel{(S 1)}{\subseteq} \mathcal{U} \cap$ $\mathscr{M} \stackrel{(S 2)}{\subseteq} \mathcal{U}, \mathscr{M} \stackrel{(S 3)}{\subseteq} \mathcal{U} \cup \mathscr{M} \stackrel{(S 4)}{\subseteq} \mathcal{U} \mathscr{M}$.

Proof. Since an ultrametric is a metric, $\mathscr{M} \mathscr{U} \subseteq \mathscr{U}$ and $\mathscr{M} \mathscr{U} \subseteq$ $\mathscr{M}$. So (S1) follows. Similarly, $\mathscr{U} \subseteq \mathcal{U} \mathscr{M}$ and $\mathscr{M} \subseteq \mathcal{U} \mathscr{M}$, so (S4) holds. (S2) and (S3) are true in general.

We will obtain characterization of the functions in $\mathcal{U}$, $\mathscr{M}$, and $\mathcal{U} \mathscr{M}$ in later section. Then we will show that the relation $\subseteq$ in Proposition 6 is in fact a proper subset. It is easy to see that if $f \in \mathscr{M}$, then $f$ is amenable. We extend this to the case of any function $f \in \mathscr{M} \cup \mathscr{M} \cup \cup \mathscr{M} \cup \mathcal{U}$.

Proposition 7. If $f \in \mathscr{U} \mathscr{M}$, then $f$ is amenable.

Proof. Assume that $f \in \mathscr{U} \mathscr{M}$. To show that $f$ is amenable, we let $x \in[0, \infty)$ be such that $f(x)=0$. Let $X=\{A, B, C\} \subseteq \mathbb{R}^{2}$, where $A=(-x / 2,0), B=(x / 2,0)$, and $C=(0, \sqrt{3} x / 2)$. Let $d_{2}$ be the Euclidean metric on $\mathbb{R}^{2}$ and let $d=\left.d_{2}\right|_{X}$ be the restriction of $d_{2}$ on $X$. Then $d(A, B)=d(A, C)=d(B, C)=x$. Therefore, $(X, d)$ is an ultrametric space. So $f \circ d$ is a metric on $X$. Now $f(0)=f(d(A, A))=(f \circ d)(A, A)=0$, and $(f \circ d)(A, B)=f(d(A, B))=f(x)=0$, which implies $A=B$. That is, $(-x / 2,0)=(x / 2,0)$. Hence $x=0$. This shows that $f$ is amenable as desired.

Corollary 8. If $f:[0, \infty) \rightarrow[0, \infty)$ is in $\mathscr{M}, \mathscr{M} \mathcal{U}, \mathcal{U}$, or $\mathcal{U} \mathscr{M}$, then $f$ is amenable.

Proof. By Proposition 6, $\mathscr{M} \cup \mathscr{M} \mathcal{U} \cup \mathcal{U} \cup \mathcal{U} \mathscr{M}=\mathscr{U} \mathscr{M}$. So the result follows from Proposition 7.

\section{Ultrametric-Preserving Functions}

In this section, we obtain characterizations of ultrametricpreserving functions. Then we compare their properties with those of metric-preserving functions.

Theorem 9. Let $f:[0, \infty) \rightarrow[0, \infty)$. Then $f$ is ultrametricpreserving if and only if $f$ is amenable and increasing. 
Proof. Assume that $f$ is ultrametric-preserving. By Corollary 8 , it suffices to show that $f$ is increasing. Let $a, b \in[0, \infty)$ and $a<b$. Let $d_{2}$ be the Euclidean metric on $\mathbb{R}^{2}$ and let $X=\{A, B, C\} \subseteq \mathbb{R}^{2}$, where $A=(-a / 2,0)$, $B=(a / 2,0)$, and $C=\left(0, \sqrt{\left(4 b^{2}-a^{2}\right) / 4}\right)$. Let $d=\left.d_{2}\right|_{X}$ be the restriction of $d_{2}$ on $X$. Then $d(A, B)=a$, $d(A, C)=d(B, C)=b$. Therefore, $(X, d)$ is an ultrametric space. Since $f$ is ultrametric-preserving, $f \circ d$ is an ultrametric. Therefore,

$$
\begin{aligned}
f(a) & =f \circ d(A, B) \\
& \leq \max \{f \circ d(A, C), f \circ d(B, C)\} \\
& =f(b),
\end{aligned}
$$

as required. Next assume that $f$ is increasing and amenable. Let $(X, d)$ be an ultrametric space, and let $x, y, z \in X$. Since $f$ is amenable, $f \circ d(x, y)=0$ if and only if $x=y$. Since $d$ is an ultrametric, $d(x, z) \leq \max \{d(x, y), d(y, z)\}$. So $d(x, z) \leq d(x, y)$ or $d(x, z) \leq d(y, z)$. If $d(x, z) \leq d(x, y)$, then $f(d(x, z)) \leq f(d(x, y)) \leq \max \{f \circ d(x, y), f \circ d(y, z)\}$. If $d(x, z) \leq d(y, z)$, then $f(d(x, z)) \leq f(d(y, z)) \leq \max \{f \circ$ $d(x, y), f \circ d(y, z)\}$. In any case $f \circ d(x, z) \leq \max \{f \circ$ $d(x, y), f \circ d(y, z)\}$. Therefore, $f \circ d$ is an ultrametric. This completes the proof.

Corollary 10. Let $f:[0, \infty) \rightarrow[0, \infty)$. Then the following statements hold:

(i) if $f$ is ultrametric-preserving and subadditive, then $f$ is metric-preserving;

(ii) if $f$ is metric-preserving and increasing on $[0, \infty)$, then $f$ is ultrametric-preserving.

Proof. We obtain that (i) follows from Theorem 9 and Lemma 2, and (ii) follows from Corollary 8 and Theorem 9.

The next example shows that $\mathscr{M} \nsubseteq \mathscr{U}$ and $\mathscr{U} \nsubseteq \mathscr{M}$.

Example 11. Let $f, g:[0, \infty) \rightarrow[0, \infty)$ be given by

$$
f(x)=x^{2}, \quad g(x)= \begin{cases}0, & \text { if } x=0 ; \\ 1, & \text { if } x \in \mathbb{Q}-\{0\} ; \\ 2, & \text { if } x \in \mathbb{Q}^{c} .\end{cases}
$$

By Theorem 9, $f$ is ultrametric-preserving and $g$ is not ultrametric-preserving. If $d$ is the usual metric on $\mathbb{R}$, we see that

$$
f \circ d(1,3)=f(2)=4>2=f \circ d(1,2)+f \circ d(2,3) .
$$

So $f \circ d$ is not a metric and therefore $f$ is not metricpreserving. Since $g(x) \in[1,2]$ for all $x>0, g$ is tightly bounded, and therefore, by Lemma $3, g$ is metric-preserving. In conclusion, $f \in \mathscr{U}, f \notin \mathscr{M}, g \in \mathscr{M}$, and $g \notin \mathscr{U}$. This shows that $\mathscr{U} \nsubseteq \mathscr{M}$ and $\mathscr{M} \nsubseteq \mathscr{U}$. This example also shows that the relations (S2) and (S3) in Proposition 6 are proper subsets.

Next we give some results concerning concavity of the functions in $\mathscr{U} \cup \mathscr{M}$.
Theorem 12. Let $f:[0, \infty) \rightarrow[0, \infty)$. If $f$ is amenable and concave, then $f$ is ultrametric-preserving.

Proof. Assume that $f$ is amenable and concave. We will show that $f$ is increasing. First observe that if $y>0$, then $f(y)>$ $f(0)$ because $f$ is amenable. Next let $y>x>0$ and suppose for a contradiction that $f(y)<f(x)$. Let $t=f(y) / f(x), x_{1}=$ $(y f(x)-x f(y)) /(f(x)-f(y))$, and $x_{2}=x$. Then $t \in(0,1)$, and $x_{1}, x_{2} \in(0, \infty)$. Since $f$ is concave, we obtain

$$
\begin{aligned}
f(y) & =f\left((1-t) x_{1}+t x_{2}\right) \\
& \geq(1-t) f\left(x_{1}\right)+t f\left(x_{2}\right) \\
& =(1-t) f\left(x_{1}\right)+f(y) .
\end{aligned}
$$

This implies that $f\left(x_{1}\right)=0$ which contradicts the fact that $x_{1}>0$ and $f$ is amenable. Hence $f$ is increasing on $[0, \infty)$. By Theorem $9, f$ is ultrametric-preserving.

Corollary 13. If $f:[0, \infty) \rightarrow[0, \infty)$ is amenable and concave, then $f$ is both ultrametric-preserving and metricpreserving.

Proof. The first part comes from Theorem 12. The other part has appeared in the literature but we will give an alternative proof here. We know that $f$ is increasing by Theorems 12 and 9. So by Lemma 2, it suffices to show that $f$ is subadditive. Let $a, b \in(0, \infty)$. By Lemma 4 , we have $f(a+b) /(a+b) \leq$ $\min \{f(a) / a, f(b) / b\}$. Therefore,

$$
\begin{aligned}
f(a+b) & =a\left(\frac{f(a+b)}{a+b}\right)+b\left(\frac{f(a+b)}{a+b}\right) \\
& \leq a \frac{f(a)}{a}+b \frac{f(b)}{b}=f(a)+f(b),
\end{aligned}
$$

as required. This completes the proof.

The next example shows that there exists a function which is both metric-preserving and ultrametric-preserving but not concave.

Example 14. Let $f:[0, \infty) \rightarrow[0, \infty)$ be defined by

$$
f(x)= \begin{cases}x, & x \in[0,1] \\ 1, & x \in[1,10] \\ x-9, & x \in(10,11) \\ 2, & x \geq 11\end{cases}
$$

It is easy to see that $f$ is amenable and increasing. So, by Theorem 9, $f$ is ultrametric-preserving. Next we will show that $f$ is metric-preserving. By Lemma 2, it suffices to show that $f$ is subadditive. Observe that $f(x) \leq x$ and $f(x) \leq 2$ for every $x \in[0, \infty)$. We consider $a, b \in[0, \infty)$ in several cases.

$$
\begin{aligned}
& \text { If } a, b \in[0,1] \text {, then } f(a)+f(b)=a+b \geq f(a+b) \text {. } \\
& \text { If } a, b \in[1,10] \text {, then } f(a)+f(b)=2 \geq f(a+b) \text {. } \\
& \text { Similarly, if } a, b>10 \text {, then } f(a)+f(b)>2 \geq f(a+b) \text {. }
\end{aligned}
$$


If $a \in[0,1], b \in[1,10]$, then

$f(a)+f(b)=a+1 \geq \max \{1, a+b-9\} \geq f(a+b)$.

If $a \in[0,1], b \in[10,11]$, then $f(a)+f(b)=a+b-9 \geq$ $f(a+b)$.

If $a \in[0,1], b \in[11, \infty)$, then $f(a)+f(b)=a+2 \geq$ $2=f(a+b)$.

If $a \in[1,10], b \in[10, \infty)$, then $f(a)+f(b)=b-8 \geq$ $2=f(a+b)$.

The other cases can be obtained similarly. Therefore, $f$ is subadditive. Hence, $f$ is metric-preserving. But $f((9+$ $11) / 2)<(f(9)+f(11)) / 2$, so $f$ is not concave. That is, $f \in$ $\mathcal{U} \cap \mathscr{M}$ but $f$ is not concave. In addition, $f$ is not a constant on $(0, \infty)$. So this example also shows that $\mathscr{U} \cap \mathscr{M} \nsubseteq \mathscr{M} \mathscr{U}$ and the relation (S1) in Proposition 6 is a proper subset.

\section{Metric-Ultrametric-Preserving Functions}

In this section, we characterize the functions in $\mathscr{M} \mathcal{U}$. We will see that this notion is so strong that it forces the functions to be a constant on $(0, \infty)$. More precisely, we obtain the following theorem.

Theorem 15. Let $f:[0, \infty) \rightarrow[0, \infty)$. Then $f$ is metricultrametric-preserving if and only if $f$ is amenable and $f$ is a constant on $(0, \infty)$.

Proof. First assume that $f$ is amenable and is a constant on $(0, \infty)$. That is there exists a constant $c>0$ such that

$$
f(x)= \begin{cases}0, & \text { if } x=0 \\ c, & \text { if } x>0\end{cases}
$$

To show that $f$ is metric-ultrametric-preserving, let $(X, d)$ be a metric space and let $x, y, z \in X$. If $x=y$ or $x=z$ or $y=z$, then it is easy to see that $f \circ d(x, y) \leq \max \{f \circ d(x, z), f \circ$ $d(z, y)\}$. If $x, y, z$ are all distinct, then $f \circ d(x, y)=c=f \circ$ $d(x, z)=f \circ d(y, z)$ and therefore

$$
f \circ d(x, y) \leq \max \{f \circ d(x, z), f \circ d(z, y)\}
$$

This shows that $f \circ d$ is an ultrametric. In the other direction, we assume that $f \in \mathscr{M} \mathcal{U}$. By Corollary 8 , it is enough to show that $f$ is a constant on $(0, \infty)$. Throughout the proof, we let $d$ be the usual metric on $\mathbb{R}$ and $d_{2}$ the Euclidean metric on $\mathbb{R}^{2}$. We will apply Lemma 5 repeatedly. First we will show that

$$
f(1)=f\left(\frac{1}{n}\right)=f\left(\frac{m}{n}\right) \quad \text { for every } m, n \in \mathbb{N} \text {. }
$$

So we let $m, n \in \mathbb{N}$ be arbitrary. Since $f \in \mathscr{M} \mathcal{U}, f \circ d$ is an ultrametric on $\mathbb{R}$. By Lemma 5 , we have

$$
\begin{aligned}
f(1)= & f \circ d(0,1) \\
\leq & \max \left\{f \circ d\left(0, \frac{1}{n}\right), f \circ d\left(\frac{1}{n}, \frac{2}{n}\right), \ldots,\right. \\
& \left.f \circ d\left(\frac{n-1}{n}, 1\right)\right\} \\
= & \max \left\{f\left(\frac{1}{n}\right), f\left(\frac{1}{n}\right), \ldots, f\left(\frac{1}{n}\right)\right\}=f\left(\frac{1}{n}\right) .
\end{aligned}
$$

Next let $A=(-1 / 2 n, 0), B=(1 / 2 n, 0), C=$ $\left(0, \sqrt{\left(4-(1 / n)^{2}\right) / 4}\right)$ be points in $\mathbb{R}^{2}$. Since $f \in \mathscr{M} \mathscr{U}, f \circ d_{2}$ is an ultrametric on $\mathbb{R}^{2}$. Therefore,

$$
\begin{aligned}
f\left(\frac{1}{n}\right) & =f \circ d_{2}(A, B) \leq \max \left\{f \circ d_{2}(A, C), f \circ d_{2}(C, B)\right\} \\
& =\max \{f(1), f(1)\}=f(1) .
\end{aligned}
$$

Therefore, $f(1)=f(1 / n)$. By a similar method, we obtain

$$
\begin{aligned}
f\left(\frac{m}{n}\right) & =f \circ d\left(0, \frac{m}{n}\right) \\
& \leq \max \left\{f \circ d\left(\frac{k-1}{n}, \frac{k}{n}\right) \mid k \in\{1,2, \ldots, m\}\right\} \\
& =f\left(\frac{1}{n}\right) .
\end{aligned}
$$

In addition, we let $A=(-1 / 2 n, 0), B=(1 / 2 n, 0), C=$ $\left(0, \sqrt{\left.\left(4(m / n)^{2}-(1 / n)^{2}\right) / 4\right)}\right.$ be points in $\mathbb{R}^{2}$ so that

$$
\begin{aligned}
f\left(\frac{1}{n}\right) & =f \circ d_{2}(A, B) \leq \max \left\{f \circ d_{2}(A, C), f \circ d_{2}(C, B)\right\} \\
& =f\left(\frac{m}{n}\right) .
\end{aligned}
$$

Therefore, $f(m / n)=f(1 / n)$. Hence $f(m / n)=f(1 / n)=f(1)$ for every $m, n \in \mathbb{N}$, as asserted. We conclude that

$$
f(q)=f(1) \quad \text { for every } x \in \mathbb{Q} \cap(0, \infty) .
$$

Next let $a \in \mathbb{Q}^{c} \cap(0, \infty)$. We will show that $f(a)=f(1)$. Let $q_{1}, q_{2} \in \mathbb{Q} \cap(0, \infty)$ be such that $q_{1}<a<q_{2}$. Let $A_{1}=\left(-q_{1} / 2,0\right), B_{1}=\left(q_{1} / 2,0\right), C_{1}=\left(0, \sqrt{\left(4 a^{2}-q_{1}^{2}\right) / 4}\right)$, $A_{2}=(-a / 2,0), B_{2}=(a / 2,0), C_{2}=\left(0, \sqrt{\left(4 q_{2}^{2}-a^{2}\right) / 4}\right)$ be points in $\mathbb{R}^{2}$. By (18) and the fact that $f \circ d_{2}$ is an ultrametric on $\mathbb{R}^{2}$, we obtain

$$
\begin{aligned}
f(1) & =f\left(q_{1}\right)=f \circ d_{2}\left(A_{1}, B_{1}\right) \\
& \leq \max \left\{f \circ d_{2}\left(A_{1}, C_{1}\right), f \circ d_{2}\left(C_{1}, B_{1}\right)\right\} \\
& =f(a)=f \circ d_{2}\left(A_{2}, B_{2}\right) \\
& \leq \max \left\{f \circ d_{2}\left(A_{2}, C_{2}\right), f \circ d_{2}\left(C_{2}, B_{2}\right)\right\} \\
& =f\left(q_{2}\right)=f(1) .
\end{aligned}
$$


This shows that

$$
f(a)=f(1) \quad \forall a \in \mathbb{Q}^{c} \cap(0, \infty) .
$$

From (18) and (20), we see that $f(x)=f(1)$ for all $x \in(0, \infty)$. This completes the proof.

Let $f$ be a metric-preserving function and let $d$ be a metric. Then either $f \circ d$ is a metric equivalent to $d$ or $f \circ d$ is a uniformly discrete metric $[3,6]$. In addition, $f$ is continuous on $[0, \infty)$ if and only if it is continuous at $0[3,4,6]$. But by Theorem 15, every metric-ultrametric-preserving function $f$ is always discontinuous at 0 and $f \circ d$ is always a uniformly discrete metric for all metric $d$. We record this in the next corollary.

Corollary 16. Let $f:[0, \infty) \rightarrow[0, \infty)$ be metricultrametric-preserving. Then

(i) $f \circ d$ is a uniformly discrete metric for every metric $d$,

(ii) $f$ is discontinuous at 0 and is continuous on $(0, \infty)$.

Proof. By Theorem 15, there exists $c>0$ such that

$$
f(x)= \begin{cases}0, & \text { if } x=0 \\ c, & \text { if } x>0\end{cases}
$$

So (ii) follows immediately. If $(X, d)$ is a metric space, then

$$
f \circ d(x, y)= \begin{cases}0, & \text { if } x=y \\ c, & \text { if } x \neq y .\end{cases}
$$

So if we let $\varepsilon=c / 2$, then $B_{f \circ d}(x, \varepsilon)=\{x\}$ for every $x \in X$. This proves (i).

\section{Ultrametric-Metric-Preserving Functions}

In this section, we give a characterization of the functions in $\mathscr{U} \mathscr{M}$ in terms of special type of triangle triplets. Recall that a triple $(a, b, c)$ of nonnegative real numbers is called triangle triplet if $a \leq b+c, b \leq c+a$, and $c \leq a+b$. We denote by $\Delta$ the set of all triangle triplets. We introduce a special type of triangle triplets that will be used to characterize ultrametricmetric-preserving functions in the next definition.

Definition 17. A triple $(a, b, c)$ of nonnegative real numbers will be called ultra-triangle triplet if $a \leq \max \{b, c\}, b \leq$ $\max \{c, a\}$, and $c \leq \max \{a, b\}$. We denote by $\Delta_{\infty}$ the set of all ultra-triangle triplets.

Since we will compare the functions $f$ in $\mathcal{U} \mathscr{M}$ with those in $\mathscr{M}$, we first state a characterization of metric-preserving functions in terms of triangle triplets.

Theorem 18. Let $f:[0, \infty) \rightarrow[0, \infty)$ be amenable. Then the following statements are equivalent:

(i) $f$ is metric-preserving,

(ii) for each $(a, b, c) \in \Delta,(f(a), f(b), f(c)) \in \Delta$,

(iii) for each $(a, b, c) \in \Delta, f(a) \leq f(b)+f(c)$.
Proof. The proof can be found, for example, in $[3,4,6]$.

Similar to Theorem 18, we obtain a characterization of the functions in $\mathscr{U} \mathscr{M}$ in terms of ultra-triangle triplets as follows.

Theorem 19. Let $f:[0, \infty) \rightarrow[0, \infty)$ be amenable. Then the following statements are equivalent:

(i) $f$ is ultrametric-metric-preserving,

(ii) for each $(a, b, c) \in \Delta_{\infty},(f(a), f(b), f(c)) \in \Delta$,

(iii) for each $0 \leq a \leq b, f(a) \leq 2 f(b)$.

To prove Theorem 19, the following lemmas are useful.

Lemma 20. If $(X, d)$ is an ultrametric space and $x, y, z \in X$, then the triple $(d(x, y), d(x, z), d(z, y))$ is an ultra-triangle triplet. Conversely, if $(a, b, c)$ is an ultra-triangle triplet, then there exist an ultrametric space $(X, d)$ and $x, y, z \in X$ such that $(a, b, c)=(d(x, y), d(x, z), d(z, y))$.

Lemma 21. If $(a, b, c) \in \Delta_{\infty}$, then
(i) $a \leq b=c$ or
(ii) $b \leq c=a$ or
(iii) $c \leq a=b$.

We will prove Lemmas 21 and 20, and then Theorem 19, respectively.

Proof of Lemma 21. Let $(a, b, c) \in \Delta_{\infty}$. Suppose that $a, b, c$ are all distinct. Without loss of generality, we can assume that $a<$ $b<c$. Then $c>\max \{a, b\}$ which contradicts the fact that $(a, b, c) \in \Delta_{\infty}$. So $a, b, c$ are not all distinct. If $a=b$, then $c \leq \max \{a, b\}=a$ and (iii) holds. Similarly, if $a=c$, then (ii) holds and if $b=c$, then (i) holds.

Proof of Lemma 20. The first part follows immediately from the ultrametric inequality of $d$. For the converse, we let $(a, b, c) \in \Delta_{\infty}$. By Lemma 21, we can assume that $a \leq$ $b=c$ (the other cases can be proved similarly). Let $X=$ $\{A, B, C\} \subseteq \mathbb{R}^{2}$, where $A=(-a / 2,0), B=(a / 2,0)$, and $C=\left(0, \sqrt{\left(4 b^{2}-a^{2}\right) / 4}\right)$. Let $d_{2}$ be the Euclidean metric on $\mathbb{R}^{2}$ and $d=\left.d_{2}\right|_{X}$. Then $(X, d)$ is an ultrametric space and $(a, b, c)=\left(d_{2}(A, B), d_{2}(A, C), d_{2}(C, B)\right)$.

Proof of Theorem 19. (i) $\rightarrow$ (ii) Let $f \in \mathcal{U} \mathscr{M}$ and let $(a, b, c) \in$ $\Delta_{\infty}$. Then by Lemma 20, there exist an ultrametric space $(X, d)$ and $x, y, z \in X$ such that

$$
(a, b, c)=(d(x, y), d(x, z), d(z, y)) .
$$

Since $f \in \mathscr{U} \mathscr{M},(X, f \circ d)$ is a metric space. It follows from the triangle inequality of $f \circ d$ that $(f \circ d(x, y), f \circ d(x, z), f \circ$ $d(z, y))$ is a triangle triplet. That is, $(f(a), f(b), f(c)) \in \Delta$.

(ii) $\rightarrow$ (iii) Assume that (ii) holds. Let $0 \leq a \leq b$. Then, $(a, b, b) \in \Delta_{\infty}$. So $(f(a), f(b), f(b)) \in \Delta$ by (ii). Therefore, $f(a) \leq f(b)+f(b)=2 f(b)$, as required.

(iii) $\rightarrow$ (i) Assume that (iii) holds. Let $(X, d)$ be an ultrametric space. Since $f$ is amenable, $f \circ d(x, y)=0$ if and only if $x=y$. So it remains to show that the triangle inequality holds for $f \circ d$. Let $x, y, z \in X$. Then by Lemma 20 , $(d(x, y), d(x, z), d(z, y)) \in \Delta_{\infty}$. Then by Lemma 21 , we can 
assume that $d(x, y) \leq d(x, z)=d(z, y)$ (the other cases can be proved similarly). Then by (iii), we obtain

$$
\begin{aligned}
f \circ d(x, y) & =f(d(x, y)) \leq 2 f(d(x, z)) \\
& =f(d(x, z))+f(d(z, y)) \\
& =f \circ d(x, z)+f \circ d(z, y), \quad \text { as required. }
\end{aligned}
$$

Hence, the proof is complete.

Next we give an example to show that the relation (S4) in Proposition 6 is a proper subset.

Example 22. Let $f:[0, \infty) \rightarrow[0, \infty)$ be given by

$$
f(x)= \begin{cases}x, & \text { if } x \leq 1 \\ \frac{1}{2}, & \text { if } x>1\end{cases}
$$

Let $d$ be the usual metric on $\mathbb{R}$. Then

$$
\begin{aligned}
f \circ d(1,2) & =f(1)=1>\frac{1}{3}+\frac{1}{2} \\
& =f \circ d\left(1, \frac{2}{3}\right)+f \circ d\left(\frac{2}{3}, 2\right) .
\end{aligned}
$$

So $f \circ d$ is not a metric and therefore $f \notin \mathscr{M}$. Since $f$ is not increasing, $f \notin \mathcal{U}$. Next we will show that $f \in \mathcal{U} \mathscr{M}$, by applying Theorem 19. Let $0 \leq a \leq b$. If $b \geq 1 / 2$, then $f(b) \geq 1 / 2$ and therefore $2 f(b) \geq 1 \geq f(x)$ for all $x \in[0, \infty)$. In particular, $2 f(b) \geq f(a)$. If $b<1 / 2$, then $a<1 / 2$ and thus $f(a)=a \leq b=f(b) \leq 2 f(b)$. In any case, we have $f(a) \leq 2 f(b)$. Hence $f \in \mathcal{U} \mathscr{M}$ but $f \notin \mathscr{M}$ and $f \notin \mathcal{U}$. This example shows that $\mathscr{U} \mathscr{M} \nsubseteq \mathscr{U} \cup \mathscr{M}$ and the relation (S4) in Proposition 6 is in fact a proper subset.

Remark 23. (1) From Examples 11, 14, and 22, we now see that the relations (S1), (S2), (S3), and (S4) in Proposition 6 are in fact proper subsets.

(2) If we replace $1 / 2$ in the definition of $f$ in Example 22 by a constant $c$ (that is, $f(x)=x$ if $x \leq 1$ and $f(x)=c$ if $x>1$ ), then $f \in \mathscr{U} \mathscr{M}$ if and only if $c \geq 1 / 2$.

\section{Continuity}

In this section, we investigate the continuity aspect of the functions in $\mathscr{M}, \mathscr{U}, \mathscr{U} \mathscr{M}$, and $\mathscr{M} \mathscr{U}$. By Corollary 16 , the continuity of metric-ultrametric-preserving functions is trivial: they are always discontinuous at 0 and continuous elsewhere. The continuity of metric-preserving functions has also been investigated by many authors $[1-4,6,8,18]$, but we can still extend it further in the next theorem.

Before we state the theorem, let us recall some definitions concerning generalized continuities. Let $f:[0, \infty) \rightarrow$ $[0, \infty)$. Then $f$ is said to be weakly continuous at $a \neq 0$ if and only if there are sequences $\left(x_{n}\right)$ and $\left(y_{n}\right)$ such that $\left(x_{n}\right)$ is strictly increasing and converges to $a,\left(y_{n}\right)$ is strictly decreasing and converges to $a$, and $f\left(x_{n}\right)$ and $f\left(y_{n}\right)$ converge to $f(a)$. If $a=0$, then $f$ is said to be weakly continuous at $a$ if and only if there exists a strictly decreasing sequence $\left(y_{n}\right)$ converging to $a$ such that $f\left(y_{n}\right)$ converges to $f(a)$. We refer the reader to [36] for weak continuity of functions defined on a more general domain.

Unlike weak continuity, quasi continuity and almost continuity seem to be first given in a more general domain than a subset of $\mathbb{R}$. So we let $X$ and $Y$ be topological spaces and let $g: X \rightarrow Y$. Then $g$ is said to be quasi continuous at $a \in X$ if for all open sets $U$ of $X$ and $V$ of $Y$ such that $a \in U$ and $f(a) \in V$, there is a nonempty open sets $G$ of $X$ such that $G \subseteq U$ and $f(G) \subseteq V$. The function $g$ is said to be almost continuous at $x$ in the sense of Singal (briefly a.c.S. at $x$ ) if for each open set $V$ of $Y$ containing $f(x)$, there exists an open set $U$ containing $x$ such that $f(U) \subseteq \operatorname{Int}(\bar{V})$ and $g$ is said to be almost continuous at $x$ in the sense of Husain (briefly a.c.H. at $x$ ) if for each open set $V$ of $Y$ containing $f(x)$, $\overline{f^{-1}(V)}$ is a neighborhood of $x$. The function $g$ is said to be quasi continuous on $A \subseteq X$ (or a.c.S. on $A$, or a.c.H. on $A$ ) if it is quasi continuous at every $a \in A$ (a.c.S. at $a$ for every $a \in A$, a.c.H. at $a$ for every $a \in A$ ).

Remark 24. (1) The concepts of a.c.S. functions and a.c.H. functions are not equivalent as shown by Long and Carnahan [37].

(2) There are several other types of continuities in the literature. Some of them have the same name but different definition, see [38] for instance, a different definition of weak continuity. We refer the reader to [39-43] and the other references for additional details and information.

Now we are ready to state our theorem. We will see that there is a similarity and dissimilarity between continuity of the functions in $\mathscr{M}$ and $\mathscr{U} \mathscr{M}$.

Theorem 25. Let $f:[0, \infty) \rightarrow[0, \infty)$ be metric-preserving. The following statements are equivalent:

(1) $f$ is continuous at $[0, \infty)$,

(2) $f$ is continuous at 0 ,

(3) For every $\varepsilon>0$, there exists and $x>0$ such that $f(x)<$ $\varepsilon$,

(4) $f$ is strongly metric-preserving,

(5) $f$ is uniformly continuous on $[0, \infty)$,

(6) $f$ is weakly continuous on $[0, \infty)$,

(7) $f$ is weakly continuous at 0 ,

(8) $f$ is quasi continuous on $[0, \infty)$,

(9) $f$ is quasi continuous at 0 ,

(10) $f$ is a.c.S on $[0, \infty)$,

(11) $f$ is a.c.S at 0 ,

(12) $f$ is a.c.H on $[0, \infty)$,

(13) $f$ is a.c.H at 0 . 
Proof. The equivalence of (1), (2), (3), and (4) is proved in $[4,6]$. With a bit more observation, we can prove that (1) to (11) are all equivalent. First we notice that

$$
|f(a)-f(b)| \leq f(|a-b|) \quad \forall a, b \in[0, \infty) .
$$

To prove (28), we let $a, b \in[0, \infty)$. Then $(a, b,|a-b|)$ is a triangle triplet. So by Theorem $18,(f(a), f(b), f(|a-b|))$ is a triangle triplet. Therefore,

$$
f(a) \leq f(b)+f(|a-b|), \quad f(b) \leq f(a)+f(|a-b|) .
$$

Thus, $|f(a)-f(b)| \leq f(|a-b|)$, as asserted. Now we will prove that (2), (5), (6), (7), and (3) are equivalent.

$(2) \rightarrow(5)$ Assume that $f$ is continuous at 0 . Let $\varepsilon>0$. Then there exists a $\delta>0$ such that

$$
\text { if } a \in[0, \delta) \text {, then } f(a)<\varepsilon \text {. }
$$

Now if $x, y \in[0, \infty)$ and $|x-y|<\delta$, then by (28) and (30), we obtain

$$
|f(x)-f(y)| \leq f(|x-y|)<\varepsilon .
$$

This shows that $f$ is uniformly continuous on $[0, \infty)$.

It is easy to see that (5) implies (6) and (6) implies (7).

$(7) \rightarrow$ (3) We assume that (7) holds. Let $\left(x_{n}\right)$ be the sequence in $(0, \infty)$ such that $\left(x_{n}\right)$ is strictly decreasing and converges to 0 , and $\left(f\left(x_{n}\right)\right)$ converges to $f(0)=0$. Therefore, if $\varepsilon>0$ is given, there exists $N \in \mathbb{N}$ such that

$$
f\left(x_{N}\right)=f\left(x_{N}\right)-f(0)<\varepsilon .
$$

This proves (3). Since (3) and (2) are equivalent, we see that (2), (5), (6), (7), and (3) are equivalent, as asserted.

It is true in general that every continuous function is quasi continuous. So it is easy to see that (1) implies (8) and (8) implies (9). Next assume that (9) holds. To show (3), let $\varepsilon>0$ be given. Let $V=U=[0, \varepsilon)$. Then $V$ and $U$ are open set in $[0, \infty)$ containing $f(0)=0$ and 0 , respectively. Since $f$ is quasi continuous at 0 , there exists a nonempty open set $G \subseteq U$ such that $f(G) \subseteq V$. Now we can choose $x \in G-\{0\}$ so that $x>0$ and $f(x)<\varepsilon$. This gives (3). Since (1) and (3) are equivalent, we obtain that (1), (8), (9), and (3) are equivalent. Similarly, it is easy to see that (1) implies (10), (10) implies (11), (1) implies (12), and (12) implies (13). Since (1) and (3) are equivalent, it now suffices to show that each of (11) and (13) implies (3). First assume that (11) holds. Let $\varepsilon>0$ and let $V=[0, \varepsilon)$. Then $V$ is open in $[0, \infty)$ and contains $f(0)$. Since $f$ is a.c.S. at 0 , there exists an open set $U$ containing 0 such that

$$
f(U) \subseteq \operatorname{Int}(\bar{V})=\operatorname{Int}[0, \varepsilon]=[0, \varepsilon)
$$

Now we can choose $x \in U-\{0\}$ so that $x>0$ and $f(x)<\varepsilon$. Similarly if (13) holds, then $\overline{f^{-1}(V)}$ is a neighborhood of 0 , so $f^{-1}(V) \neq\{0\}$, and therefore we can choose $x \in f^{-1}(V)-\{0\}$ so that $f(x)<\varepsilon$ and $x>0$. This completes the proof.
The function $f$ in Example 22 shows that in the case of ultrametric-metric-preserving functions, the global continuity on $[0, \infty)$ and the local continuity at 0 are not equivalent. In addition, the uniform continuity on $[0, \infty)$ and continuity on $[0, \infty)$ are not equivalent as can be seen from the function $f$ in Example 11. However, we still have the following result for the continuity at 0 .

Theorem 26. Let $f$ be ultrametric-metric-preserving. Then the following statements are equivalent:

(i) $f$ is continuous at 0 ,

(ii) $f$ is weakly continuous at 0 ,

(iii) for every $\varepsilon>0$, there exists an $x>0$ such that $f(x)<\varepsilon$,

(iv) $f$ is quasi continuous at 0 ,

(v) $f$ is a.c.S. at 0 ,

(vi) $f$ is a.c.H. at 0 .

Proof. We have that (i) implies (ii) is true in general. By the same argument that (7) implies (3) in Theorem 25, we see that (ii) implies (iii). Next assume that (iii) holds. To show that $f$ is continuous at 0 , let $\varepsilon>0$ be given. Then by (iii), there exists $x_{0}>0$ such that $f\left(x_{0}\right)<\varepsilon / 2$. Let $\delta=x_{0}$ and let $x \in[0, \delta)$. Since $0 \leq x<\delta$ and $f \in \mathcal{U} \mathscr{M}$, we obtain by Corollary 8 and Theorem 19 that

$$
|f(x)-f(0)|=f(x) \leq 2 f(\delta)=2 f\left(x_{0}\right)<\varepsilon .
$$

This gives (i). Therefore, (i), (ii), and (iii) are equivalent. Since (i) implies (iv), (v), and (vi), it suffices to show that each of (iv), (v), and (vi) implies (iii). Since $f \in \mathscr{U} \mathscr{M}$, it is amenable and we can use the same argument of the proof of Theorem 25 to show that (iv) implies (iii) (the same as (9) implies (3)), (v) implies (iii) (the same as (11) implies (3)), and (vi) implies (iii) (the same as (13) implies (3)). This completes the proof.

Corollary 27. Let $f \in \mathcal{U} \mathscr{M}$. If $f$ is discontinuous at 0 , then there exists an $\varepsilon>0$ such that $f(x)>\varepsilon$ for all $x>0$.

Proof. This follows from (i) and (iii) in Theorem 26.

Example 28. Let $f, g:[0, \infty) \rightarrow[0, \infty)$ be given by

$$
\begin{gathered}
f(x)= \begin{cases}x, & x \leq 1 ; \\
1, & x>1, \quad x \in \mathbb{Q} ; \\
2, & x>1, \quad x \notin \mathbb{Q},\end{cases} \\
g(x)= \begin{cases}x, & x<1 ; \\
2, & x \geq 1\end{cases}
\end{gathered}
$$

First we will show that $f \in \mathscr{U} \mathscr{M}$ by applying Theorem 19 . So we let $0 \leq a \leq b$. If $b>1$, then $2 f(b) \geq 2 \geq f(x)$ for every $x \in[0, \infty)$. In particular, $2 f(b) \geq f(a)$. If $b \leq 1$, then $f(a)=a \leq b \leq 2 b=2 f(b)$. So $f \in \mathcal{U} \mathscr{M}$. It is easy to see that $f$ is weakly continuous at 1 but is not continuous at 1. In fact $f$ is weakly continuous at every $x \geq 0$ and is not continuous at any $x \geq 1$. This shows that we cannot replace 
continuity at 0 in Theorem 26 by continuity at any other point $x \neq 0$. Similarly, $g \in \mathscr{U} \mathscr{M}$ and is quasi continuous on $[0, \infty)$ but $g$ is not continuous at 1 .

\section{Conflict of Interests}

The authors declare that they have no competing interests.

\section{Acknowledgment}

The first author received financial support from The Thailand Research Fund (research Grant no. TRG5680052). The author takes this opportunity to thank The Thailand Research Fund for the support.

\section{References}

[1] W. A. Wilson, "On certain types of continuous transformations of metric spaces," American Journal of Mathematics, vol. 57, no. 1, pp. 62-68, 1935.

[2] J. Borsík and J. Doboš, "On metric preserving functions," Real Analysis Exchange, vol. 13, no. 1, pp. 285-293, 1988.

[3] Y. Borsik and J. Doboš, "Functions whose composition with every metric is a metric," Mathematica Slovaca, vol. 31, no. 1, pp. 3-12, 1981.

[4] P. Corazza, "Introduction to metric-preserving functions," The American Mathematical Monthly, vol. 106, no. 4, pp. 309-323, 1999.

[5] P. P. Das, "Metricity preserving transforms," Pattern Recognition Letters, vol. 10, no. 2, pp. 73-76, 1989.

[6] J. Doboš, "Metric Preserving Functions," http://web.science .upjs.sk/jozefdobos/wpcontent/uploads/2012/03/mpf1.pdf.

[7] J. Doboš, "On modifications of the Euclidean metric on reals," Tatra Mountains Mathematical Publications, vol. 8, pp. 51-54, 1996.

[8] J. Doboš, "A survey of metric preserving functions," Questions and Answers in General Topology, vol. 13, no. 2, pp. 129-134, 1995.

[9] J. Doboš and Z. Piotrowski, "When distance means money," International Journal of Mathematical Education in Science and Technology, vol. 28, pp. 513-518, 1997.

[10] J. Doboš and Z. Piotrowski, "A note on metric preserving functions," International Journal of Mathematics and Mathematical Sciences, vol. 19, no. 1, pp. 199-200, 1996.

[11] J. Doboš and Z. Piotrowski, "Some remarks on metric preserving functions," Real Analysis Exchange, vol. 19, no. 1, pp. 317-320, 1994.

[12] J. Kelly, General Topology, Springer, 1955.

[13] A. Petruşel, I. A. Rus, and M.-A. Şerban, "The role of equivalent metrics in fixed point theory," Topological Methods in Nonlinear Analysis, vol. 41, no. 1, pp. 85-112, 2013.

[14] Z. Piotrowski and R. W. Vallin, "Functions which preserve Lebesgue spaces," Commentationes Mathematicae: Prace Matematyczne, vol. 43, no. 2, pp. 249-255, 2003.

[15] I. Pokorný, "Some remarks on metric preserving functions," Tatra Mountains Mathematical Publications, vol. 2, pp. 65-68, 1993.

[16] T. K. Sreenivasan, "Some properties of distance functions," The Journal of the Indian Mathematical Society, vol. 11, pp. 38-43, 1947.
[17] I. Termwuttipong and P. Oudkam, "Total boundedness, completeness and uniform limits of metric-preserving functions," Italian Journal of Pure and Applied Mathematics, no. 18, pp. 187196, 2005.

[18] R. W. Vallin, "Continuity and differentiability aspects of metric preserving functions," Real Analysis Exchange, vol. 25, no. 2, pp. 849-868, 1999/00.

[19] K. Włodarczyk and R. Plebaniak, "Contractions of Banach, Tarafdar, Meir-Keeler, Ćirić-Jachymski-Matkowski and Suzuki types and fixed points in uniform spaces with generalized pseudodistances," Journal of Mathematical Analysis and Applications, vol. 404, no. 2, pp. 338-350, 2013.

[20] K. Włodarczyk and R. Plebaniak, "Leader type contractions, periodic and fixed points and new completivity in quasi-gauge spaces with generalized quasi-pseudodistances," Topology and Its Applications, vol. 159, no. 16, pp. 3504-3512, 2012.

[21] O. Kada, T. Suzuki, and W. Takahashi, "Nonconvex minimization theorems and fixed point theorems in complete metric spaces," Mathematica Japonica, vol. 44, no. 2, pp. 381-391, 1996.

[22] T. Khemaratchatakumthorn and I. Termwuttipong, "Metricpreserving functions, $w$-distances and Cauchy $w$-distances," Thai Journal of Mathematics, vol. 5, no. 3, pp. 51-56, 2007.

[23] J. Marín, S. Romaguera, and P. Tirado, "Weakly contractive multivalued maps and $w$-distances on complete quasi-metric spaces," Fixed Point Theory and Applications, vol. 2011, article 2, 2011.

[24] T. Suzuki, " $w$-distances and $\tau$-distances," Nonlinear Functional Analysis and Applications, vol. 13, no. 1, pp. 15-27, 2008.

[25] T. Suzuki, “On Downing-Kirk's theorem," Journal of Mathematical Analysis and Applications, vol. 286, no. 2, pp. 453-458, 2003.

[26] T. Suzuki, "Generalized distance and existence theorems in complete metric spaces," Journal of Mathematical Analysis and Applications, vol. 253, no. 2, pp. 440-458, 2001.

[27] T. Suzuki and W. Takahashi, "Fixed point theorems and characterizations of metric completeness," Topological Methods in Nonlinear Analysis, vol. 8, no. 2, pp. 371-382, 1996.

[28] S. Bosch, U. Güntzer, and R. Remmert, Non-Archimedean Analysis, vol. 261 of Grundlehren der Mathematischen Wissenschaften, Springer, 1984.

[29] R. Deepa, P. N. Natarajan, and V. Srinivasan, "Cauchy multiplication of Euler summable series in ultrametric fields," Commentationes Mathematicae, vol. 53, no. 1, pp. 73-79, 2013.

[30] M. Cencelj, D. Repovš, and M. Zarichnyi, "Max-min measures on ultrametric spaces," Topology and Its Applications, vol. 160, no. 5, pp. 673-681, 2013.

[31] K. Funano, "Two infinite versions of the nonlinear Dvoretzky theorem," Pacific Journal of Mathematics, vol. 259, no. 1, pp. 101108, 2012.

[32] M. Mendel and A. Naor, "Ultrametric subsets with large Hausdorff dimension," Inventiones Mathematicae, vol. 192, no. 1, pp. 1-54, 2013.

[33] E. Yurova, "On ergodicity of $p$-adic dynamical systems for arbitrary prime $p$, , p-Adic Numbers, Ultrametric Analysis, and Applications, vol. 5, no. 3, pp. 239-241, 2013.

[34] J. P. Coleman, "Nonexpansive algebras," Algebra Universalis, vol. 55, no. 4, pp. 479-494, 2006.

[35] S. Priess-Crampe and P. Ribenboim, "Ultrametric spaces and logic programming," Journal of Logic Programming, vol. 42, no. 2, pp. 59-70, 2000.

[36] P. Pongsriiam, T. Khemaratchatakumthorn, I. Termwuttipong, and N. Triphop, "On weak continuity of functions," Thai Journal of Mathematics, vol. 3, no. 1, pp. 7-16, 2005. 
[37] P. E. Long and D. A. Carnahan, "Comparing almost continuous functions," Proceedings of the American Mathematical Society, vol. 38, pp. 413-418, 1973.

[38] N. Levine, "A decomposition of continuity in topological spaces," The American Mathematical Monthly, vol. 68, pp. 4446, 1961.

[39] K. Ciesielski and A. Rosłanowski, "Two examples concerning almost continuous functions," Topology and Its Applications, vol. 103, no. 2, pp. 187-202, 2000.

[40] J. Ewert, "Characterization of cliquish functions," Acta Mathematica Hungarica, vol. 89, no. 4, pp. 269-276, 2000.

[41] O. V. Maslyuchenko, "The discontinuity point sets of quasicontinuous functions," Bulletin of the Australian Mathematical Society, vol. 75, no. 3, pp. 373-379, 2007.

[42] T. Noiri and V. Popa, "On some forms of weakly continuous functions in bitopological spaces," Demonstratio Mathematica, vol. 41, no. 3, pp. 685-700, 2008.

[43] R. J. Pawlak, A. Loranty, and A. Bąkowska, "On the topological entropy of continuous and almost continuous functions," Topology and Its Applications, vol. 158, no. 15, pp. 2022-2033, 2011. 


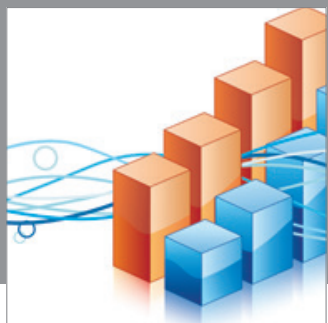

Advances in

Operations Research

mansans

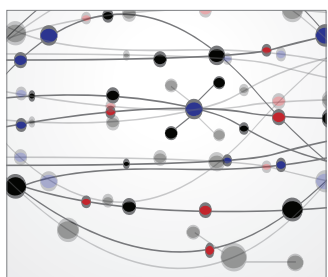

The Scientific World Journal
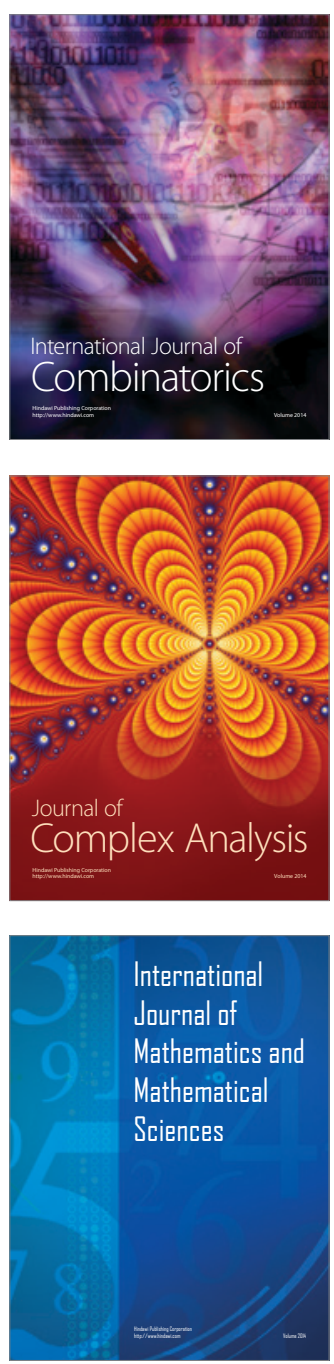
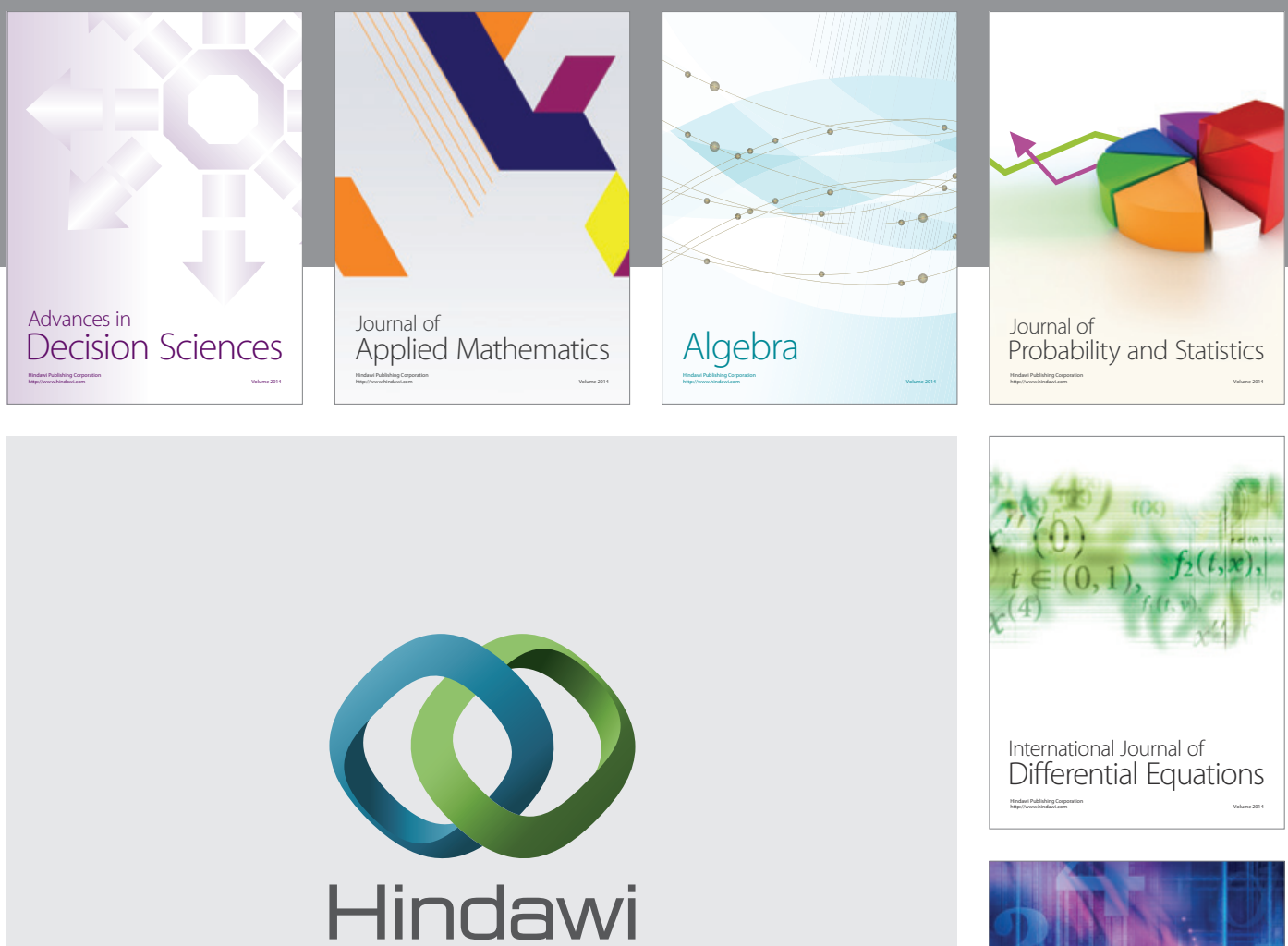

Submit your manuscripts at http://www.hindawi.com
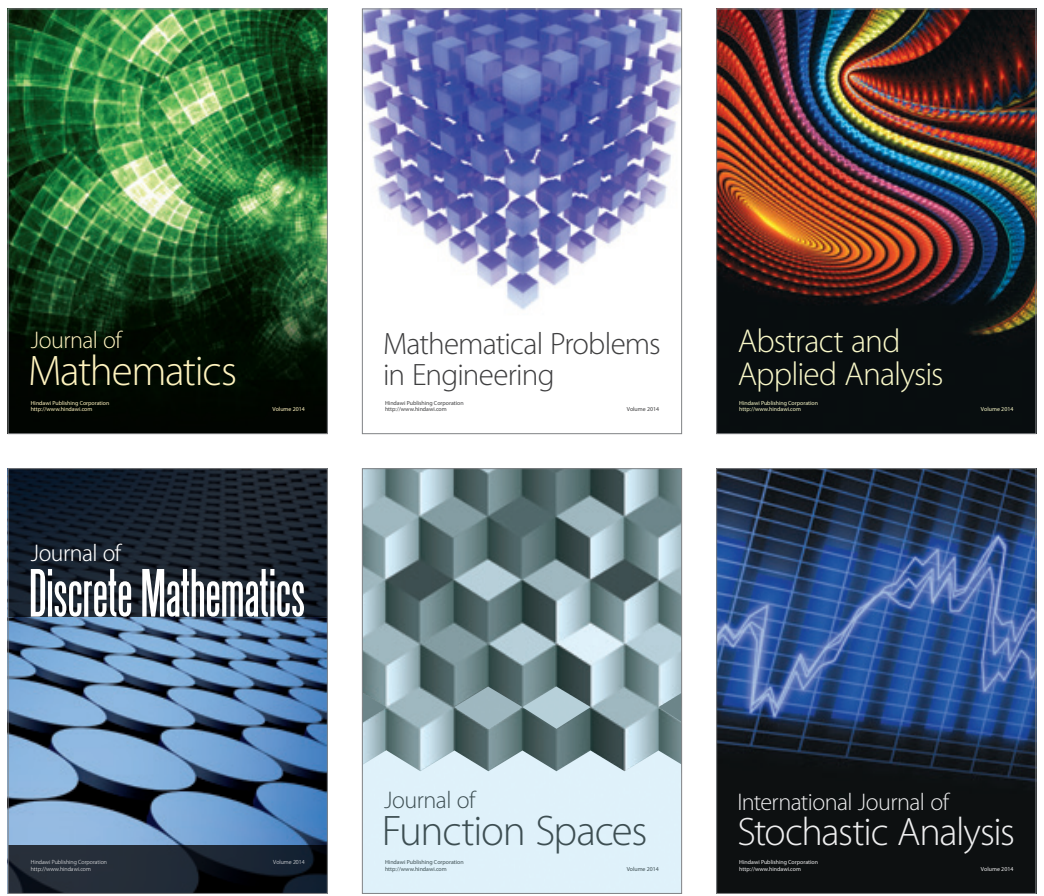

Journal of

Function Spaces

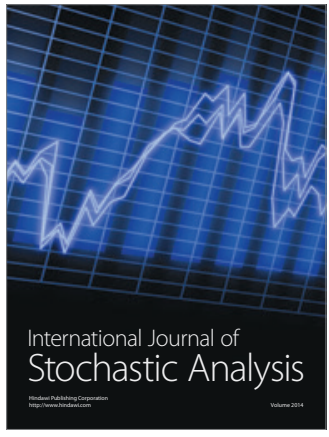

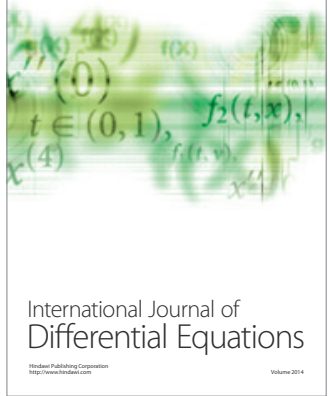
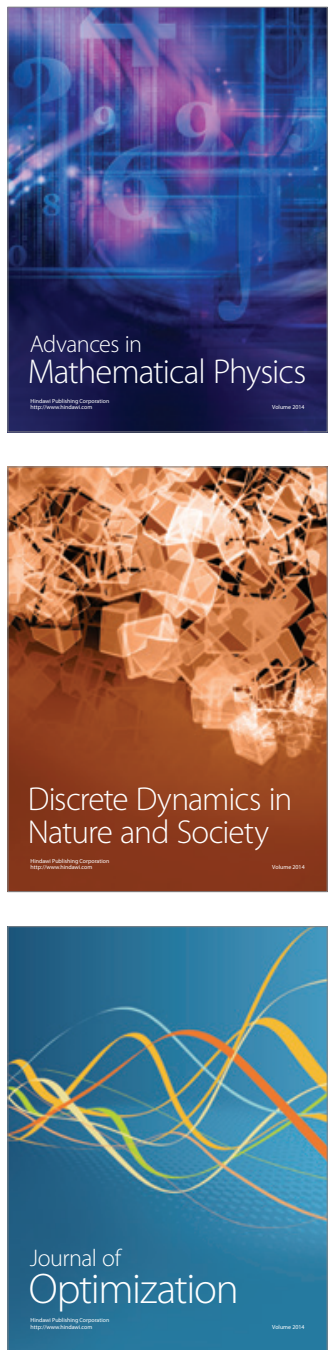